Military Technical College

Kobry Elkobbah,

Cairo, Egypt

May 27-29,2008

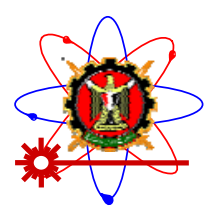

$4^{\text {th }}$ International Conference on Mathematics and Engineering Physics (ICMEP-4)

EM-3

\title{
HEAT TRANSFER FOR HERSCHEL-BULKLEY FLUIDS IN THE ENTRANCE REGION OF A RECTANGULAR DUCT
}

\author{
SAYED-AHMED* M. E. AND KISHK* KAREM M.
}

\begin{abstract}
In this study, we present a numerical solution for combined laminar fluid flow and heat transfer of Herschel-Bulkley non-Newtonian fluids in the entrance region of a rectangular duct. The governing equations are solved iteratively by using finite difference method to obtain temperature, bulk temperature, and Nusselt number. Two cases of the thermal boundary conditions are considered; (i) $T$ thermal boundary condition "constant temperature at the wall" and (ii) $\mathrm{H} 2$ thermal boundary condition "constant heat flux at the wall". The results are presented in Tables and Figures for different parameters for the fluid and the duct geometry.
\end{abstract}

\section{KEY WORDS}

Entrance Region flow, Heat transfer, Non-Newtonian fluids, Numerical analysis, and Rectangular duct.

*Professor, Dpt. of Engineering Mathematics and Physics, Fayoum University, Egypt. *Assistant lecturer, Dpt. of Engineering Mathematics and Physics, Fayoum University, Egypt. 


\section{NOMENCLATURE}

$\begin{array}{ll}a & \text { half major side of the duct } \\ b & \text { half minor side of the duct } \\ c & \text { specific heat } \\ D_{h} & \text { hydraulic diameter of the duct } \\ G_{Z} & \text { Graetz number } \\ h & \text { convective heat transfer coefficient } \\ K & \text { thermal conductivity } \\ k & \text { consistency index } \\ M & \text { number of mesh intervals in the cross- section of the duct } \\ N & \text { outer normal coordinate at a point on the duct wall inside periphery } \\ N u_{x, H 2} & \text { Local Nusselt number for the }(H 2) \text { boundary condition } \\ N u_{x, T} & \text { Local Nusselt number for the }(T) \text { boundary condition } \\ n & \text { flow index of the model } \\ P_{\mathrm{e}} & \text { entrance axial pressure } \\ P_{r} & \text { Prandtl number } \\ q " & \text { heat flux per unit area of the duct } \\ T_{W} & \text { uniform temperature at the wall } \\ (U, V, W) & \text { dimensionless velocity component in }(X, Y, Z) \text { directions respectively } \\ x_{h} & \text { dimensionless axial coordinate for hydrodynamic entrance region } \\ x^{*} & \text { dimensionless axial coordinate for thermal entrance region } \\ \theta & \text { dimensionless temperature } \\ \theta_{b} & \text { dimensionless fluid bulk mean temperature } \\ \theta_{c} & \text { dimensionless central temperature } \\ \theta_{w, m} & \text { dimensionless mean temperature at the wall } \\ \alpha & \text { aspect ratio } \\ \mu_{r} & \text { reference viscosity } \\ \bar{\mu} & \text { dimensionless apparent viscosity } \\ \tau_{0} & \text { yield stress } \\ \tau_{D} & \text { dimensionless yield stress }\end{array}$

\section{INTRODUCTION}

The problem of fluid flow and heat transfer of non- Newtonian fluids in the entrance region of a rectangular duct is of special interest because of their wide applications in compact heat exchangers such as chemical and food processing. Most of the purely viscous non- Newtonian fluids found in industrial practice are almost highly viscous. They are therefore, often processed in the laminar flow regime. In the entrance region the hydrodynamic and thermal boundary layers develop simultaneously, this results in better heat transfer performance. This work makes a contribution to entrance heat transfer for Herschel-Bulkley fluids flowing in a rectangular duct. Fluid flow and heat transfer calculations in the entrance region have generally required approximations to overcome the nonlinearity of the differential equations.

For Newtonian fluid, Montgomery and Wibulswas [1] obtained the combined hydrodynamic and thermal entry length solutions for rectangular ducts of aspect ratios $1,0.5,1 / 3,1 / 4$, and 1/6. Shah and London [2] and Shah and Bhatti [3] have published extensive compilations of the available information of simultaneously 
developing steady laminar flow and heat transfer for flows through ducts of circular and non-circular cross-sections. Neti and Eichhorn [4] used a control volume finite difference method and marching technique to study the flow and heat transfer through the entrance region of a square duct for $P_{r}=6$. A finite element procedure for the prediction of laminar forced convection in three-dimensional parabolic flow has been presented by Nonino et. Al. [5]. In this paper, thermally and hydrodynamically developing flows in flat channels and simultaneously developing flows in the square duct for Newtonian fluid are studied.

For non-Newtonian fluids, Chandrupatla and Sastri [6] analyzed the thermal entrance length problem for a square duct for shear thinning power law fluids neglecting viscous dissipation effects. In excellent review, Hartnet and Kostic [7] have summarized the numerous investigations of heat transfer for Newtonian and nonNewtonian fluids flowing through rectangular ducts and parallel plates. Lawal and Mujumdar [8] have published an extensive literature review for non-Newtonian fluids flowing through non-circular ducts. The problem of simultaneously developing flow and heat transfer for power law fluid flowing in rectangular ducts was considered by Etemad et. Al. [9]. Etemad and Mujumdar [10] presented a numerical simulation using the Galerkin finite element method to solve the full three dimensional governing equations for steady simultaneously developing laminar power-law fluid flow and heat transfer. They studied the problem in the entrance region of a rectangular duct. The Galerkin finite element method has been used by Etemad [11] to solve governing equations for laminar power law fluid flow and heat transfer in the entrance region of cross-shaped duct. Sayed-Ahmed [12] introduced a numerical solution for laminar heat transfer of a Herschel-Bulkley fluid in the entrance region of a square duct assuming fully developed velocity profile. He solved the energy equation with dissipation effects using an implicit Crank-Nicolson method.

\section{FORMULATION OF THE PROBLEM}

The duct configuration and boundary conditions are shown in Fig.1. Both flow and heat transfer developed simultaneously from the entrance of the duct. Assuming steady laminar flow of an incompressible Herschel-Bulkley non-Newtonian fluid with constant physical properties in the entrance region of a rectangular duct. The fluid enters the duct at uniform temperature $T_{e}$, velocity $u_{e}$ and pressure $p_{e}$. The no-slip condition is applied at the duct walls and two cases of boundary conditions (uniform wall temperature everywhere $(T)$ and uniform heat flux axially as well as peripherally (H2) as thermal boundary conditions) are assumed. The velocity components $u, v$, and $w$, pressure $p$ and the temperature $T$ are developing simultaneously. Due to symmetry, the results are computed in one quadrant of the duct cross-section ( starting from the origin ).

The governing equations of Herschel-Bulkley fluid flow using boundary layer assumptions, introduced by Prandtl in 1904, are given by

$$
\begin{aligned}
& \frac{\partial u}{\partial x}+\frac{\partial v}{\partial y}+\frac{\partial w}{\partial z}=0 \\
& \rho\left(u \frac{\partial u}{\partial x}+v \frac{\partial u}{\partial y}+w \frac{\partial u}{\partial z}\right)=-\frac{\partial p}{\partial x}+\frac{\partial}{\partial y}\left(\mu \frac{\partial u}{\partial y}\right)+\frac{\partial}{\partial z}\left(\mu \frac{\partial u}{\partial z}\right) \\
& (a-y) w=(b-z) v
\end{aligned}
$$


$\int_{0}^{b} \int_{0}^{a} u d y d z=a b u_{e}$

The apparent viscosity, $\mu$ for Herschel-Bulkley fluids is given by

$\mu=\tau_{0} / \sqrt{\left(\frac{\partial u}{\partial y}\right)^{2}+\left(\frac{\partial u}{\partial z}\right)^{2}}+k\left(\sqrt{\left(\frac{\partial u}{\partial y}\right)^{2}+\left(\frac{\partial u}{\partial z}\right)^{2}}\right)^{n-1}$

The initial and boundary conditions are written as

$$
\begin{aligned}
& u(0, y, z)=u_{e}, p(0)=p_{e}, v(0, y, z)=w(0, y, z)=0 \\
& u(x, 0, z)=u(x, y, 0)=v(x, 0, z)=v(x, y, 0)=w(x, 0, z)=w(x, y, 0)=0 \\
& \left.\frac{\partial u}{\partial y}\right|_{(x, a, z)}=\left.\frac{\partial u}{\partial z}\right|_{(x, y, b)}=\left.\frac{\partial w}{\partial y}\right|_{(x, a, z)}=\left.\frac{\partial v}{\partial z}\right|_{(x, y, b)}=0, \quad v(x, a, z)=w(x, y, b)=0
\end{aligned}
$$

The energy equation ( approximated using order of magnitude analysis) with negligible viscous dissipation effect may be written as

$$
u \frac{\partial T}{\partial x}+v \frac{\partial T}{\partial y}+w \frac{\partial T}{\partial z}=(K / \rho c)\left(\frac{\partial^{2} T}{\partial y^{2}}+\frac{\partial^{2} T}{\partial z^{2}}\right)
$$

The thermal initial and boundary conditions of the problem for the two cases shown in Fig. 1 are given by:

Case (i): $T(0, y, z)=T_{e}, T(x, 0, z)=T(x, y, 0)=T_{w}$

On the symmetry axes: $\left.\frac{\partial T}{\partial y}\right|_{(x, a, z)}=\left.\frac{\partial T}{\partial z}\right|_{(x, y, b)}=0$

Case (ii): $T(0, y, z)=\left.T_{e} \frac{\partial T}{\partial y}\right|_{(x, 0, z)}=\left.\frac{\partial T}{\partial z}\right|_{(x, y, 0)}=-\frac{q^{\prime \prime}}{K}$

On the symmetry axes: $\left.\frac{\partial T}{\partial y}\right|_{(x, a, z)}=\left.\frac{\partial T}{\partial z}\right|_{(x, y, b)}=0$

Define the following non-dimensional variables and parameters $X=\frac{x}{D_{h}}, Y=\frac{y}{D_{h}}, Z=\frac{z}{D_{h}}, x_{h}=\frac{x}{D_{h} R_{e}}, U=\frac{u}{u_{e}}, V=\frac{v}{u_{e}}, W=\frac{w}{u_{e}}$, $P=\frac{p-p_{e}}{\rho u_{e}^{2}}, \quad R_{e}=\frac{\rho D_{h}^{n} u_{e}^{2-n}}{k}, \quad \tau_{D}=\frac{\tau_{0}}{k\left(u_{e} / D_{h}\right)^{n}}$ and $\alpha=\frac{b}{a}$

In addition to the non-dimensional variables, which are introduced above, we may introduce: $x^{*}=\frac{x}{D_{h} R_{e} P_{r}}, G_{Z}=1 / x^{*}, P_{r}=\frac{\mu_{r} c}{K} \quad, \theta=\frac{T-T_{w}}{T_{e}-T_{w}}$ (case (i)) and $\theta=\frac{T-T_{e}}{D_{h} q^{\prime \prime} / K}\left(\right.$ case (ii)), where, $\mu_{r}=k\left(\frac{u_{e}}{D_{h}}\right)^{(n-1)}$

Equations (1-5) in dimensionless form are written as

$$
\begin{aligned}
& \frac{\partial U}{\partial X}+\frac{\partial V}{\partial Y}+\frac{\partial W}{\partial Z}=0 \\
& U \frac{\partial U}{\partial X}+V \frac{\partial U}{\partial Y}+W \frac{\partial U}{\partial Z}=-\frac{d P}{d X}+\frac{1}{R_{e}} \frac{\partial}{\partial Y}\left(\bar{\mu} \frac{\partial U}{\partial Y}\right)+\frac{1}{R_{e}} \frac{\partial}{\partial Z}\left(\bar{\mu} \frac{\partial U}{\partial Z}\right)
\end{aligned}
$$




$$
\begin{aligned}
& \int_{0}^{(\alpha+1) / 4} \int_{0}^{(\alpha+1) / 4 \alpha} U d Y d Z=\frac{(\alpha+1)^{2}}{16 \alpha} \\
& \left(\frac{1+\alpha}{4 \alpha}-Y\right) W=\left(\frac{1+\alpha}{4}-Z\right) V
\end{aligned}
$$

where, $\bar{\mu}=\tau_{D} / \sqrt{\left(\frac{\partial U}{\partial Y}\right)^{2}+\left(\frac{\partial U}{\partial Z}\right)^{2}}+\left(\sqrt{\left(\frac{\partial U}{\partial Y}\right)^{2}+\left(\frac{\partial U}{\partial Z}\right)^{2}}\right)^{n-1}$

The initial and boundary conditions $(6)$ in the dimensionless form are given by

$$
U(0, Y, Z)=1, P(0)=0, V(0, Y, Z)=W(0, Y, Z)=0
$$

$U(X, 0, Z)=U(X, Y, 0)=V(X, 0, Z)=V(X, Y, 0)=W(X, 0, Z)=W(X, Y, 0)=0$

$\left.\frac{\partial U}{\partial Y}\right|_{(X,(\alpha+1) / 4 \alpha, Z)}=\left.\frac{\partial U}{\partial Z}\right|_{(X, Y,(\alpha+1) / 4)}=\left.\frac{\partial W}{\partial Y}\right|_{(X,(\alpha+1) / 4 \alpha, Z)}=\left.\frac{\partial V}{\partial Z}\right|_{(X, Y,(\alpha+1) / 4)}=0$

$V(X,(\alpha+1) / 4 \alpha, Z)=W(X, Y,(\alpha+1) / 4)=0$

The energy equation (7) is written in the following non-dimensional form:

$$
U \frac{\partial \theta}{\partial X}+V \frac{\partial \theta}{\partial Y}+W \frac{\partial \theta}{\partial Z}=\frac{1}{R_{e} P_{r}}\left(\frac{\partial^{2} \theta}{\partial Y^{2}}+\frac{\partial^{2} \theta}{\partial Z^{2}}\right)
$$

The thermal initial and boundary conditions (8-9) are written in the following nondimensional forms:

(a) Case (i): $\theta(0, Y, Z)=1.0$ and $\theta(X, 0, Z)=\theta(X, Y, 0)=0$

On the symmetry axes: $\left.\frac{\partial \theta}{\partial Y}\right|_{(X,(\alpha+1) / 4 \alpha, Z)}=\left.\frac{\partial \theta}{\partial Z}\right|_{(X, Y,(\alpha+1) / 4)}=0$

(b) Case (ii): $\theta(0, Y, Z)=0$ and $\left.\frac{\partial \theta}{\partial Y}\right|_{(X, 0, Z)}=\left.\frac{\partial \theta}{\partial Z}\right|_{(X, Y, 0)}=-1$

$$
\text { On the symmetry axes: }\left.\frac{\partial \theta}{\partial Y}\right|_{(X,(\alpha+1) / 4 \alpha, Z)}=\left.\frac{\partial \theta}{\partial Z}\right|_{(X, Y,(\alpha+1) / 4)}=0
$$

The local Nusselt numbers $N u_{x, T}$ and $N u_{x, H 2}$ according to the two cases of boundary conditions case (i) and case (ii) respectively are written in the following:

$$
\begin{aligned}
& N u_{x, T}=\frac{1}{\theta_{b}}\left(\left.\int_{0}^{(1+\alpha) / 4} \frac{\partial \theta}{\partial Y}\right|_{(X, 0, Z)} d Z+\left.\int_{0}^{(1+\alpha) / 4 \alpha} \frac{\partial \theta}{\partial Z}\right|_{(X, Y, 0)} d Y\right) \\
& N u_{x, H 2}=\frac{1}{\left(\theta_{w, m}-\theta_{b}\right)}
\end{aligned}
$$

Where, $\theta_{b}$ and $\theta_{w, m}$ are given by

$$
\begin{aligned}
\theta_{b} & =\int_{0}^{(1+\alpha) / 4(1+\alpha) / 4 \alpha} \int_{0}^{(1+\alpha) / 4 \alpha} U \theta d Y d Z \\
\theta_{w, m} & =\frac{4 \alpha}{(\alpha+1)^{2}}\left(\int_{0}^{(1+\alpha) / 4} \theta(X, Y, 0) d Y+\int_{0}^{(1+\alpha, 0, Z) d Z)} \theta(X)\right.
\end{aligned}
$$

\section{NUMERICAL SOLUTION}

The governing equations (10-14) and the energy equation (16) are solved simultaneously by the finite difference method at the nodes of the three-dimensional 
rectangular mesh. These equations are non-linear partial differential equations with boundary conditions (15), (17) and (18). The indexes $(i, j, k)$ indicate positions in the $X, Y, Z$ directions, respectively. The origin is designated by $i=j=k=1$. The axial mesh spacing is $\Delta X$. The cross-section centerlines, $Y=(1+\alpha) / 4 \alpha$ and, $Z=(1+\alpha) / 4$ are designated by $j=M+1$ and $k=M+1$, respectively. The difference schemes for governing equations (10-14) with boundary conditions (15) are introduced in Sayed-Ahmed and Kishk [13]. In the energy equation (16) we may use central differences for the first derivatives in $Y$ and $Z$ directions and backward difference in $X$ direction and the Crank-Nicolson finite difference method for the second order derivatives [14]. Then, the finite difference form equation (16) is written as

$$
\begin{aligned}
& U 2(j, k) \frac{\theta 2(j, k)-\theta 1(, j, k)}{\Delta X}+V 2(j, k) \frac{\theta 2(j+1, k)-\theta 2(j-1, k)}{2 \Delta Y}+ \\
& W 2(j, k) \frac{\theta 2(j, k+1)-\theta 2(j, k-1)}{2 \Delta Z} \\
& =\frac{1}{R_{e} P_{r}}\left(\frac{\theta 2(j+1, k)-2 \theta 2(j, k)+\theta 2(j-1, k)}{\Delta Y^{2}}+\frac{\theta 2(j, k+1)-2 \theta 2(j, k)+\theta 2(j, k-1)}{\Delta Z^{2}}\right)
\end{aligned}
$$

where the notation $\beta 2(j, k)$ designates the variables $U, V, W$, and $\theta$ at a point in the mesh $(i, j, k)$ and $\beta 1(j, k)$ to designate the same variables at a point in the mesh $(i-1, j, k)$.

We may use finite-difference forms for the normal first derivatives in the boundary conditions equations (17b) and (18) that introduced by Vemuri and Karplus [15]. To compute the local Nusselt numbers $N u_{x, T}$ and $N u_{x, H 2}$, we may introduce finite difference forms for the integral equations (19), (21) and (22) using Simpson's 1/3 rules for double and single integrals (Jain et al [16] ).

The governing equations (10-14) that introduced by Sayed-Ahmed and Kishk [13] and the energy equation (16) are solved by marching procedure. For each axial position, the momentum and continuity equations are first solved to obtain $U 2, V 2$, and $W 2$ [13], then equation (23) is solved to obtain $\theta 2$ where, $U 2, V 2$, and $W 2$ are considered known. The system of linearized equations (23) are solved using Successive Under-Relaxation method. The criteria of convergence $\left|U 2_{i, j}{ }^{\text {new }}-U 2_{i, j}{ }^{\text {old }}\right| \leq 10^{-7}$ for velocity and the criteria of convergence $\left|\theta 2_{i, j}{ }^{\text {new }}-\theta 2_{i, j}{ }^{\text {old }}\right| \leq 10^{-7}$ for temperature are satisfied. This process is repeated at a new axial position using values of the velocity and temperature components at the previous axial position as initial conditions, and so on, up to reach the fully developed flow and heat transfer. The results are obtained at $M=40$ and $R_{e}=500$. The truncation error of the difference schemes of the governing equations is $\mathrm{O}\left(\Delta X+\Delta Y^{2}+\Delta Z^{2}\right)$. Stability and rate of convergence are functions of the relaxation parameter, the mesh size (axial as well as transverse), and the axial velocity and temperature profiles ( Chandrupatla and Sastri [6] ).Thus the difference equations tend to the partial differential equations as $\Delta X, \Delta Y^{2}$ and $\Delta Z^{2}$ tend to zero. The basic axial mesh size used was $\Delta x^{*}=.0005$; however, axial steps as small as $10^{-5}$ were used near the duct entrance because of the high velocity and Nusselt numbers gradients in that region. The previous results indicate that the finite difference equations of the model are consistent and stable. 


\section{RESULTS AND DISCUSSIONS}

The results of the velocity components and friction factor and the pressure are discussed in [13].

Fig. 2(a-c) shows the development of the axial temperature along the axial centerline of duct $\theta_{\mathrm{C}}$ along the centerline of the duct $(Y=(1+\alpha) / 4 \alpha, Z=(1+\alpha) / 4$ ) for case (i) at the following parameters; flow index $n=0.5,1$ and 1.5 , yield stress $\tau D$ $=0$ and 0.6 , aspect ratio $\alpha=0.5$ and Prandtl number $\operatorname{Pr}=0.1$ and 10 . It is found that the axial temperature $\theta_{\mathrm{c}}$ increases with increasing the flow index $\mathrm{n}$ and the Prandtl number Pr. This means that the thermal diffusivity of the fluid increases for the decreasing of the Prandtl number Pr. It is also found that the axial temperature $\theta_{\mathrm{C}}$ decreases with increasing $\tau D$ for $n=0.5$, (but there is no significant variation at $n=1$, 1.5).

Fig. 3(a-c) shows the development of the axial temperature along the axial centerline of duct $\theta_{\mathrm{C}}$ along the centerline of the duct $(Y=(1+\alpha) / 4 \alpha, Z=(1+\alpha) / 4)$ for case (ii) for flow index $n=0.5,1$ and 1.5 , yield stress $\tau D=0$ and 0.6 , aspect ratio $\alpha=0.5$ and Prandtl number $\operatorname{Pr}=0.1$ and 10 . The centerline axia temperature $\theta_{\mathrm{c}}$ increases from zero (the initial dimensionless entrance temperature) to the fully developed profile at downstream $\left(\partial \theta / \partial x^{*}\right.$ is constant). It is found that the axial temperature $\theta_{\mathbf{C}}$ decreases with increasing the flow index $\mathrm{n}$ and the Prandtl number Pr. It is also found that the axial temperature $\theta_{c}$ increases with increasing the yield stress $\tau D$ at $n=0.5$ (but there is no significant variation at $n=1,1.5$ ).

Fig. 4(a-c) shows the temperature profiles on the central plane $\theta\left(x^{*}, Y,(\alpha+1) / 4\right)$ for case (i). The results are obtained for thermal axial position $x^{*}=.05, .1$ and 0.2 , flow index $\mathrm{n}=0.5,1$ and 1.5, yield stress $\tau \mathrm{D}=0$ and 0.6 , aspect ratio $\alpha=0.5$ and Prandtl number $\operatorname{Pr}=0.1$ and 10 . It is found that the central plane temperature $\theta\left(x^{*}, Y,(\alpha+1) / 4\right)$ increases from the value of zero (at the wall) to its local maximum value (at the axial centerline of the duct), at a certain axial position $\mathrm{x}^{*}$. It has been found that the central plane temperature $\theta\left(x^{*}, Y,(\alpha+1) / 4\right)$ increases with increasing the flow index $\mathrm{n}$, the Prandtl number $\mathrm{Pr}$ and the aspect ratio $\alpha$ but it decreases with increasing the thermal axial position $x^{*}$ and the yield stress $\tau D$ at $n=0.5$ (but there is no significant variation at $n=1,1.5)$.

Fig. 5(a-c) shows the temperature profiles on the central plane $\theta\left(x^{*}, Y,(\alpha+1) / 4\right)$ for case (ii). The results are obtained for the following parameters: thermal axial position $\mathrm{x}^{*}=.05, .1$ and 0.2 , flow index $\mathrm{n}=0.5,1$ and 1.5 , yield stress $\tau \mathrm{D}=0$ and 0.6 , aspect ratio $\alpha=0.5$ and Prandtl number $\operatorname{Pr}=0.1$ and 10 . It is found that the central plane temperature $\theta\left(x^{*}, Y,(\alpha+1) / 4\right)$ increases ( at the wall ) with increasing the flow index $\mathrm{n}$ and the Prandtl number $\operatorname{Pr}$ but this is reversed at the centerline of the duct. It is also found that the central plane temperature $\theta\left(x^{*}, Y,(\alpha+1) / 4\right)$ decreases with increasing thermal axial position $x^{*}$ and it is also decreases ( at the wall ) with increasing the yield stress $\tau D$, at $n=0.5$ (but there is no significant variation at $n=1,1.5$ ), but this is reversed at the centerline of the duct.

Figs. $6(\mathrm{a}-\mathrm{c})$ and $7(\mathrm{a}-\mathrm{c})$ show the variation of the local Nusselt number Nux, $\mathrm{T}$ with the Graetz number GZ for case (i) at the following parameters: flow index $n=0.5,1$ and 1.5 , yield stress $\tau \mathrm{D}=0$ and 0.6 , aspect ratio $\alpha=0.5$ and 1 and Prandtl number $\operatorname{Pr}=$ 0.1 and 10. It is observed that, the local Nusselt number Nux,T increases with increasing the Graetz number $\mathrm{GZ}$, but it decreases with increasing the Prandtl 
number $\operatorname{Pr}$, the flow index $\mathrm{n}$ and the aspect ratio $\alpha$. There is no significant variation in Nux, T with increasing $\tau \mathrm{D}$ from 0 to 0.6 . It has been also found that the results of this study are in good agreement with the previous results.

Figs. $8(\mathrm{a}-\mathrm{c})$ and $9(\mathrm{a}-\mathrm{c})$ show the variation of the local Nusselt number Nux,H2 with the Graetz number GZ for case (ii) at the following parameters; flow index $n=0.5,1$ and 1.5, yield stress $\tau \mathrm{D}=0$ and 0.6 , aspect ratio $\alpha=0.5$ and 1 and Prandtl number $\operatorname{Pr}=0.1$ and 10. It is observed that, the local Nusselt number Nux, $\mathrm{H} 2$ increases with increasing the Graetz number GZ, but it decreases with increasing the Prandtl number $\mathrm{Pr}$, the flow index $\mathrm{n}$ and the aspect ratio $\alpha$. There is no significant variation in Nux, $\mathrm{H} 2$ with increasing $\tau \mathrm{D}$ from 0 to 0.6 . It has been also found that the results of this study are in good agreement with the previous results.

Fig. $10(a-c)$ shows the variation of the bulk mean temperature $\theta$ b with the Graetz number $G Z$ for case (i). The results are obtained for the following parameters; flow index $\mathrm{n}=0.5,1$ and 1.5 , yield stress $\tau \mathrm{D}=0$ and 0.6 , aspect ratio $\alpha=0.5$ and Prandtl number $\operatorname{Pr}=0.1$ and 10 . It is observed that the bulk mean temperature $\theta$ b increases with increasing the Graetz number $\mathrm{GZ}$, the flow index $\mathrm{n}$ and the aspect ratio $\alpha$, but it decreases with increasing the Prandtl number Pr. There is no significant variation in $\theta$ b with increasing $\tau \mathrm{D}$ from 0 to 0.6 .

Fig. 11(a-c) shows the variation of the bulk mean temperature $\theta$ b with the Graetz number $\mathrm{GZ}$ case (ii). It is found that the bulk mean temperature $\theta_{\mathrm{b}}$ decreases with increasing the Graetz number $\mathrm{GZ}$, but it is not affected by the other parameters $(n, \alpha$, $\tau \mathrm{D}$ and $\mathrm{Pr}$ ). This can be explained by the fact that the heat flux is the same for different fluid and geometry parameters, and it is also, the same, for different Prandtl numbers. Thus, the difference in velocity profiles for different fluid flow parameters is not reflected in the bulk temperature, and the difference in temperature profiles for different Prandtl numbers is also not affected in the bulk mean temperature.

\section{CONCLUSIONS}

A finite difference method is used to solve three-dimensional parabolic equations of combined and steady laminar fluid flow and heat transfer in the entrance region of a rectangular duct. Newtonian and non-Newtonian fluids (Herschel-Bulkley fluids) are used to characterize the fluid behavior. Two cases of thermal boundary conditions ( $T$ and $H_{2}$ ) are studied. Two-dimensional storage and marching technique with a relaxation method line by line solution procedure for the difference equations are some important features of the program. The effects of the parameters (aspect ratio $\alpha$, flow index $n$, yield stress $\tau_{D}$, Prandtl number $P_{r}$, and thermal axial position $x^{*}$ ), velocities $U, V, W$ and the pressure $P$ on the temperature $\theta$, the bulk mean temperature $\theta_{b}$ and Nusselt numbers $N u_{x, T}$ and $N u_{x, H 2}$ are studied. It has been observed that the increasing of the thermal axial position $x^{*}$ decreased the axial temperature $\theta_{\mathrm{c}}$ along the centerline of the duct and the central plane temperature $\theta\left(x^{*}, Y,(\alpha+1) / 4\right)$ for case(i) but they are increases with increasing $x^{*}$ for case(ii) . It has been found that the increasing of the Graetz number $G_{Z}$ increased the local Nusselt number $N u_{x, T}$ and the bulk mean temperature $\theta_{b}$ for case(i). It also, increased the local Nusselt number $N u_{x, H 2}$ but it decreased the bulk mean temperature $\theta_{b}$ for case(ii). It has been found that the increasing of the yield stress $\tau_{D}$ decreases the axial temperature $\theta_{\mathrm{c}}$ along the centerline of the duct and the central plane temperature $\theta\left(x^{*}, Y,(\alpha+1) / 4\right)$ for case(i)(without significant variation at $n=1$, $1.5)$, but, they are increases with increasing $\tau_{D}$ for case(ii) along the centerline of the 
duct (without significant variation at $n=1,1.5)$. There are no significant variations in $N u_{x, T}, N u_{x, H 2}$ and $\theta_{b}$ with increasing $\tau_{D}$ from 0 to 0.6 . It has been found that the increasing of the flow index $n$ increased the axial temperature $\theta_{\mathrm{c}}, \theta\left(x^{*}, Y,(\alpha+1) / 4\right)$, $\theta_{b}$, but, it decreased $N u_{x, T}$ for case(i). It has been also found that the increasing of the flow index $n$ decreased the axial temperature, $\theta_{\mathrm{c}}, \theta\left(x^{*}, Y,(\alpha+1) / 4\right)$ along the centerline of the duct and $N u_{x, H 2}$ for case(ii). It has been observed that the increasing of the Prandtl number $P_{r}$ increased the axial temperature $\theta_{\mathrm{c}}$, the central plane temperature $\theta\left(x^{*}, Y,(\alpha+1) / 4\right)$ and the bulk mean temperature $\theta_{b}$, but, it decreased the local Nusselt number $N u_{x, T}$ case(i). It has been also found that the increasing of the Prandtl number $P_{r}$ decreased the axial temperature, $\theta_{c}$, the central plane temperature $\theta\left(x^{*}, Y,(\alpha+1) / 4\right)$ along the centerline of the duct and the local Nusselt number $N u_{x, H 2}$ for case(ii). It has been observed that the increasing of the aspect ratio $\alpha$ increased the axial temperature $\theta_{\mathrm{c}}$, the central plane temperature $\theta\left(x^{*}, Y,(\alpha+1) / 4\right)$ and the bulk mean temperature $\theta_{b}$, but, it decreased the local Nusselt number $N u_{x, T}$ case(i). It has been also observed that the increasing of the aspect ratio $\alpha$ decreased the axial temperature $\theta_{\mathrm{c}}$, the central plane temperature $\theta\left(x^{*}, Y,(\alpha+1) / 4\right)$ and the local Nusselt number $N u_{x, H 2}$ for case(ii). The work has shown that the bulk mean temperature $\theta_{b}$ is not affected by the flow index $n$, and, it is also, not affected by the Prandtl number $P_{r}$. The favorable comparison of the present results with previous experimental data as well as analytical and numerical results supports the accuracy of the present results.

\section{REFERENCES}

[1] Montgomery, S.R., and Wibulswas, P., Laminar Flow Heat Transfer in Ducts of Rectangular Cross-Section, In Proceeding of The Third International Heat Conference AICHE, New York, No.1, pp 85-98, (1966).

[2] Shah, R.K. and London, A.L., Laminar Flow Forced Convection Heat Transfer and Flow Friction in Straight and Curved Ducts- A Summary of Analytic Solution, TR No.75 Dep. Mech. Eng. Stanford University Stanford California, (1978).

[3] Shah, R.K. and Bhatti, M.S., Laminar Convection in Ducts, In Handbook of Single-Phase Convective Heat Transfer, ed. Kakac, S., Shah, R.k. \& Aung, p. 3.1, John Wiley \& Sons, (1987).

[4] Neti, S. and Eichhorn, R., Combined Hydrodynamic and Thermal Development in a Square Duct, Numerical Heat Transfer, No.6, pp 497-510, (1983).

[5] Nonino, C., Del Giudice, S. and Comini, G., Laminar Forced Convection in Three-Dimensional Duct Flows, Numerical Heat Transfer, No.13, pp 451-466, (1988).

[6] Chandrupatla, A.R. and Sastri, V.M.K., Laminar Forced Convection Heat Transfer of a Non-Newtonian Fluid in a Square Duct, Int. J. Heat Mass Transfer No. 20, pp 1315-1324, (1977).

[7] Hartnett, J.P. and Kostic, M., Heat Transfer to Newtonian and Non Newtonian Fluids in Rectangular Ducts, in Advances in Heat Transfer ed. Hartnett, T.F. and Irvine, Jr., p. 247 Academic Press, New York, (1989).

[8] Lawal, A. and Mujumdar, A.S., Laminar Duct Flow and Heat Transfer to Purely Viscous Non-Newtonian Fluids, Advances In Transport Processes ed. by Mujumdar A.S., and Mashelkar, R.A., p.352, Wiley Eastern, New Delhi, (1989).

[9] Etemad, S.Gh., Mujumdar A.S. and Huang, B., Viscous Dissipation Effect in Entrance Region Heat Transfer for Power Law Fluid Flowing Between Parallel Plates, Int. J. Heat and Fluid Flow, No.15(2), pp 122-131, (1994).

[10] Etemad, S.Gh., and Mujumdar, A.S., The Effect of Aspect Ratio and Rounded 
Corners on the Laminar Forced Convection Heat Transfer of a Non Newtonian Fluid in the Entrance Region of a Rectangular Duct, Int. Communications in Heat and Mass Transfer No.21(2), pp 283-396, (1994).

[11] Etemad, S.Gh., Three-Dimensional Laminar Non-Newtonian Fluid Flow and Heat Transfer in the Entrance Region of a Cross-shaped duct, Int. Comm. Heat Transfer,No. 24(7), pp 965-976, (1997).

[12] Sayed-Ahmed, M.E., Laminar Heat Transfer for Thermally Developing Flow of a Herschel-Bulkley Fluid in a Square Duct, Int. Comm. Heat Transfer, No.27(7), pp 1013-1024, (2000).

[13] Sayed-Ahmed, M.E. and Kishk, Karem M., Three Dimensional Laminar Flow of Herschel-Bulkley Fluids in The Entrance Region of A Rectangular Duct, AlAzhar University Engineering Journal, JAUES, No.2(11), pp 17-31, (2007).

[14] Mitchell, A.R. and Griffiths, D.F., The Finite Difference Method in Partial Differential Equations, John Wiley and Sons, New York, (1980).

[15] Vemuri, V., and Karplus, W.J., Digital Computer Treatment of Partial Differential Equations, Prentice-Hall Inc. Englewood Cliffs. New Jersey, (1981).

[16] Jain, M.K., Lyengar, S.R.K. and Jain, R.K., Numerical Method for Scientific and Engineering Computation, Wiely Eastern Limited New Delhi Second Edition, pp 227-235, (1991).

[17] Chndrupatla, A.R., Analytical and Experimental studies of Flow and Heat Transfer of a Non-Newtonian Fluid in a Square Duct, Ph.D Thesis Indian Institute of Technology., Madras India, (1977).

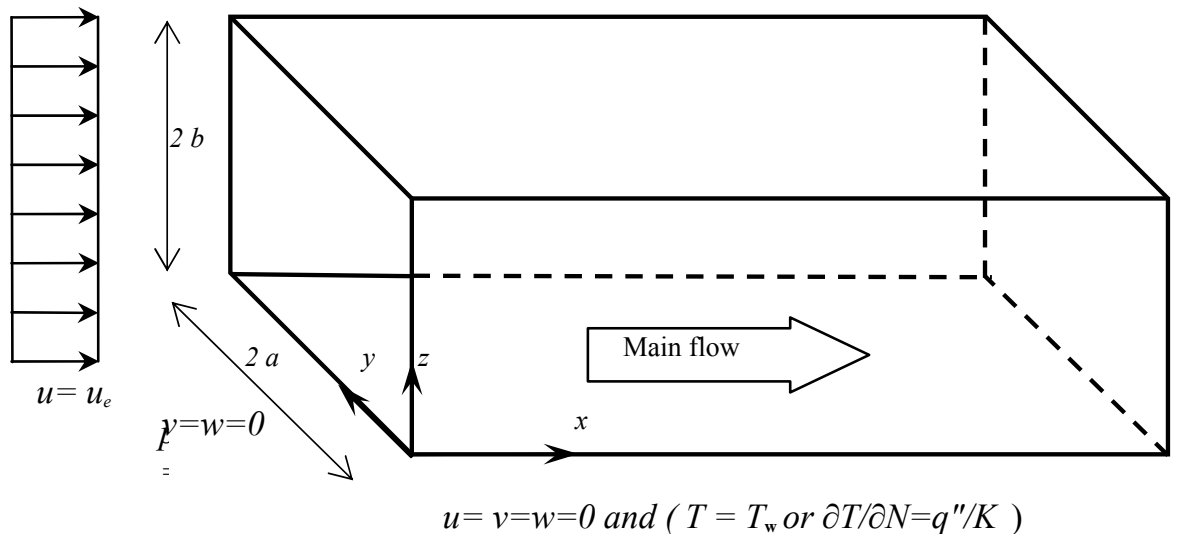

Fig.1. Duct Configuration and boundary conditions 


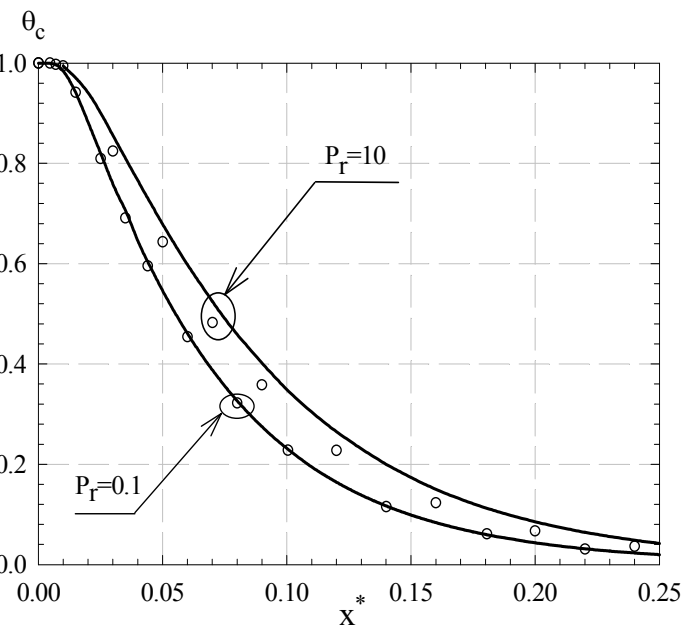

(a) $n=0.5$

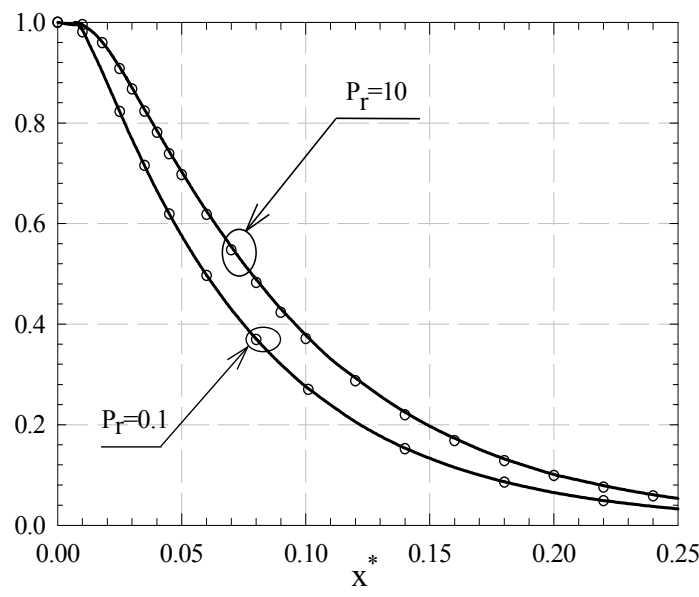

(b) $n=1$

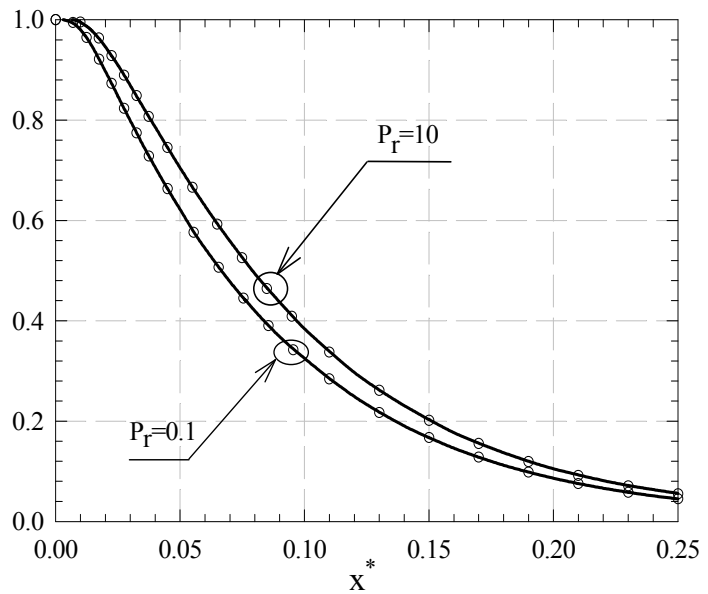

(c) $n=1.5$

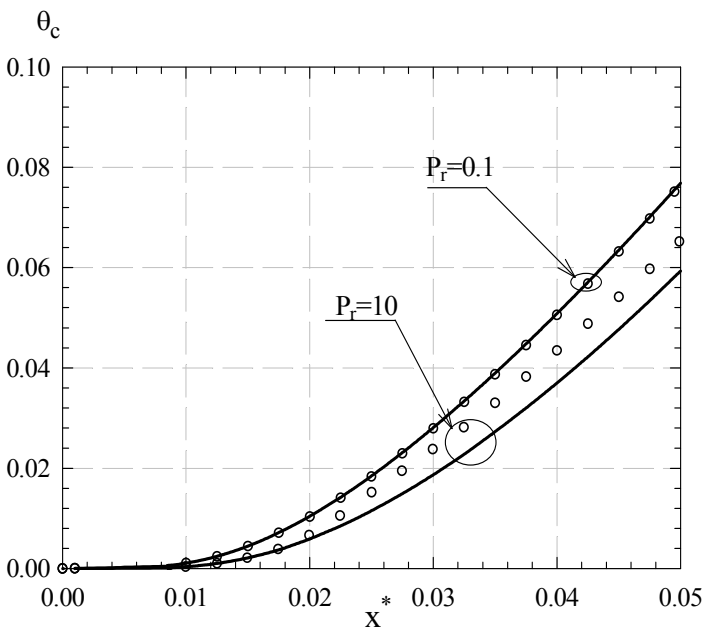

(a) $n=0.5$

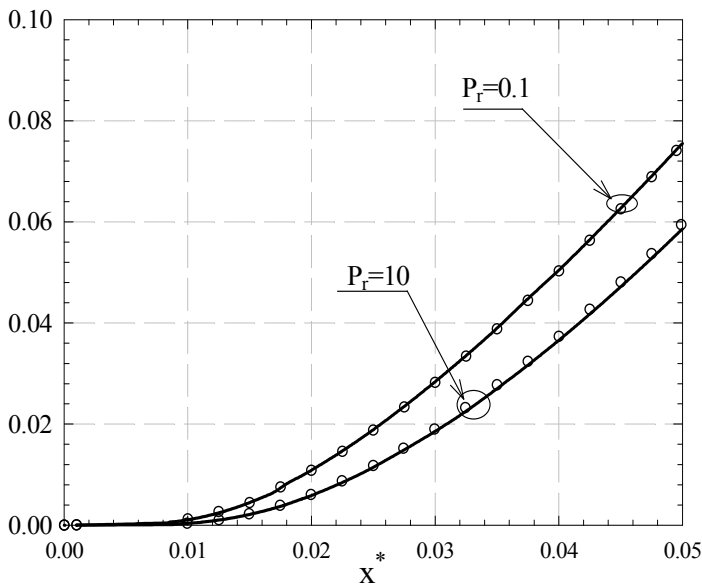

(b) $n=1$

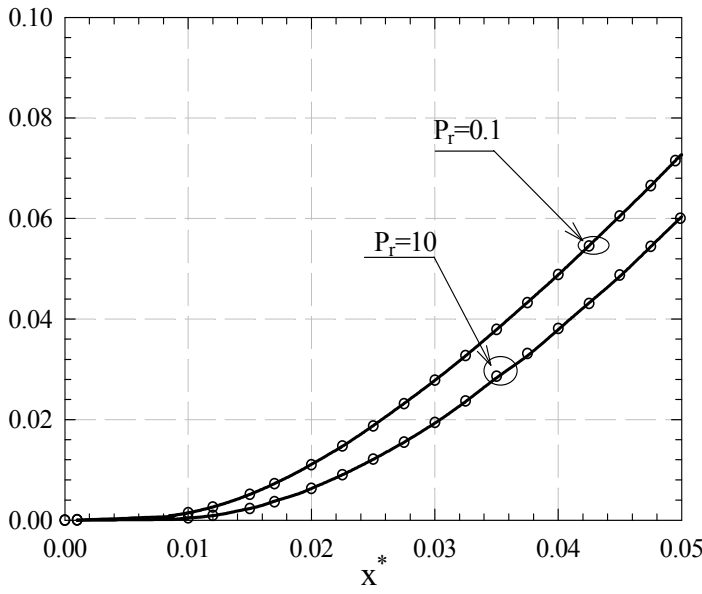

(c) $n=1.5$
Fig.2. The temperature developing along the centerline of the duct for case (i), $\alpha=0.5$
Fig.3. The temperature developing along the centerline of the duct for case (ii), $\alpha=0.5$ 

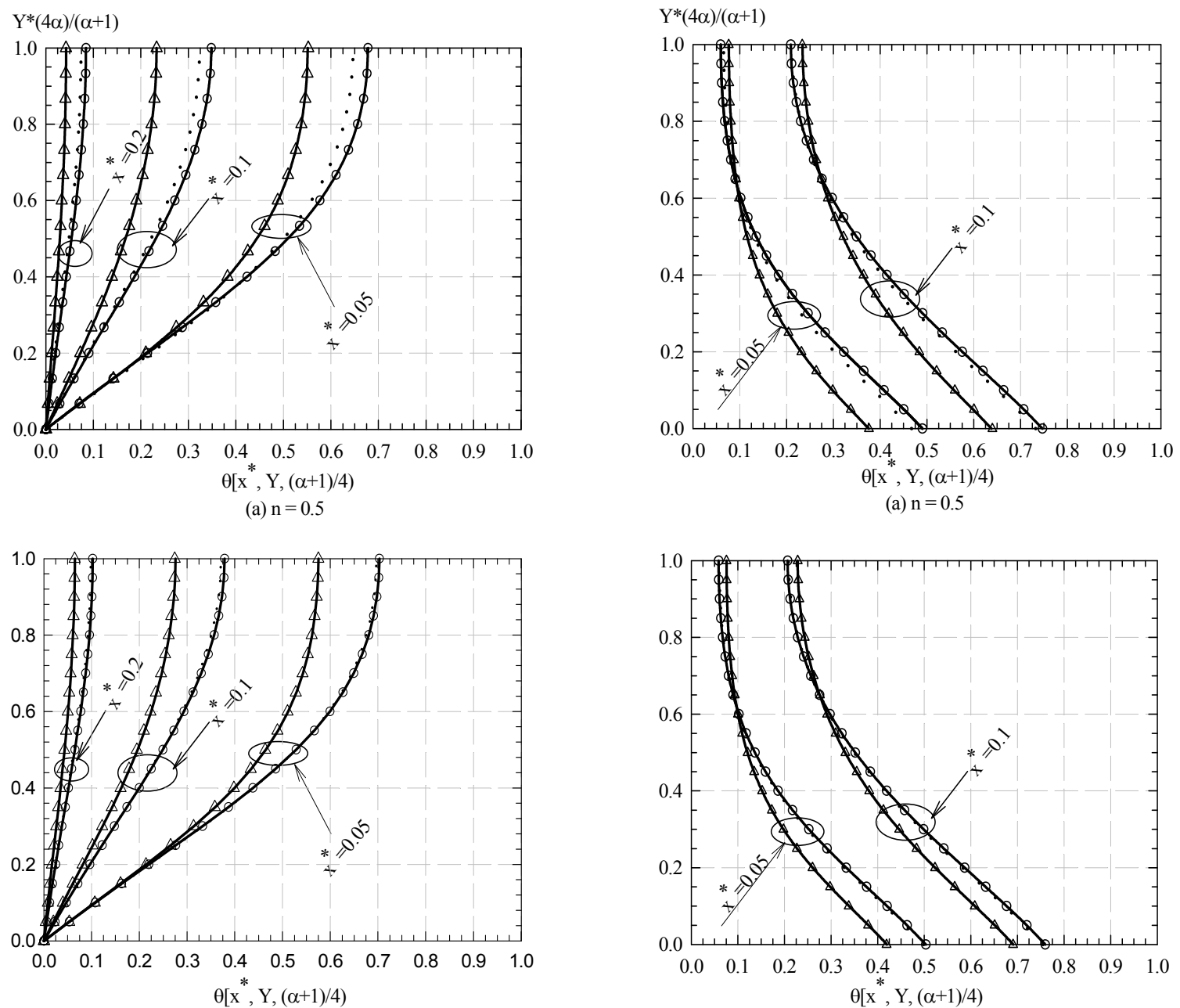

(a) $\mathrm{n}=0.5$

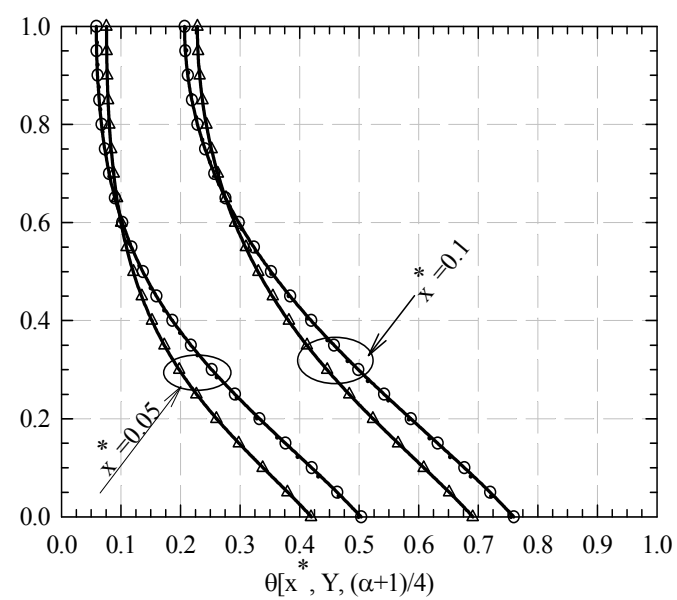

(b) $n=1$

(b) $\mathrm{n}=1$
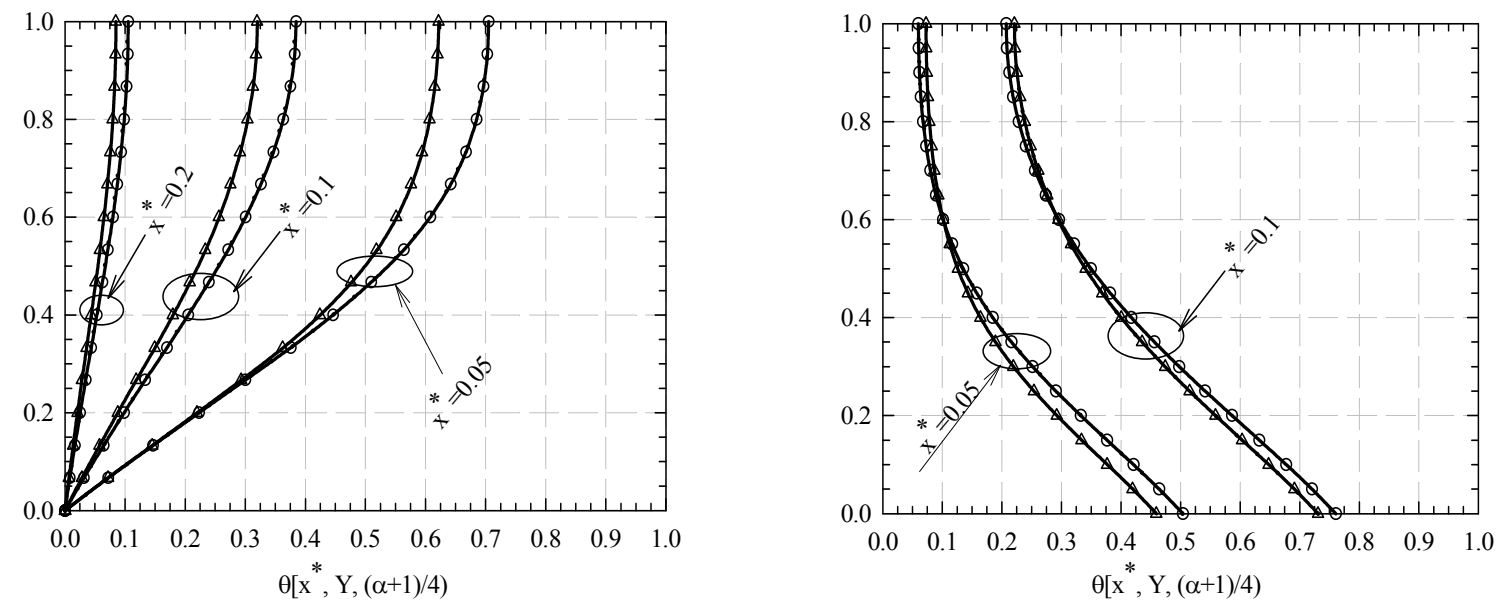

$$
\text { (c) } \left.\begin{array}{rl}
\mathrm{n}=1.5 & \longrightarrow \mathrm{P}_{\mathrm{r}}=0.1 \\
\mathrm{P}_{\mathrm{r}}=10
\end{array}\right\} \tau_{\mathrm{D}=0}
$$$$
\left.\begin{array}{cc}
\Delta & \mathrm{P}_{\mathrm{r}}=0.1 \\
\ldots & \mathrm{P}_{\mathrm{r}}=10
\end{array}\right\} \tau_{\mathrm{D}=0.6}
$$

(c) $\mathrm{n}=1.5$

Fig.4. The temperature profiles at centerline of the duct cross-section at different axial positions $x^{*}$ for case (i), $\alpha=0.5$
Fig.5. The temperature profiles at centerline of the duct cross-section at different axial positions $x^{*}$ for case (ii), $\alpha=0.5$ 


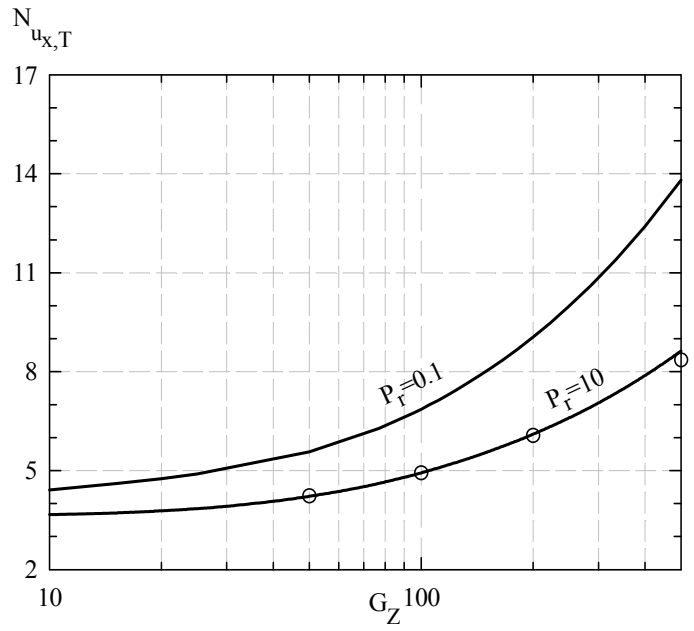

(a) $\mathrm{n}=0.5$

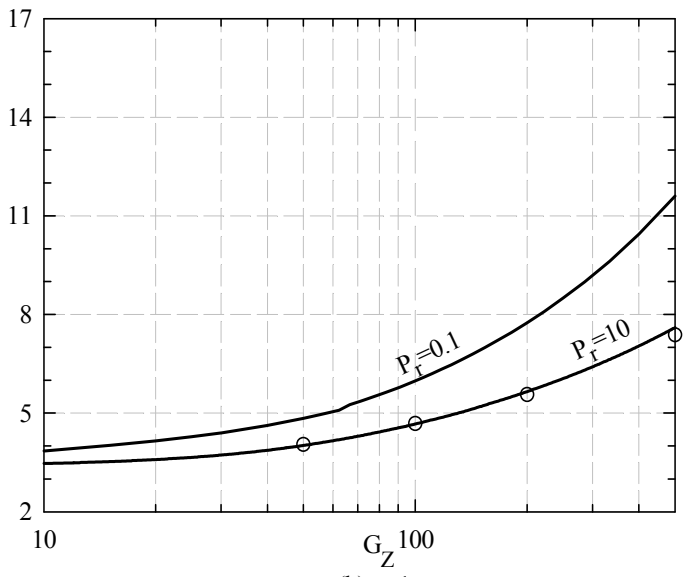

(b) $\mathrm{n}=1$

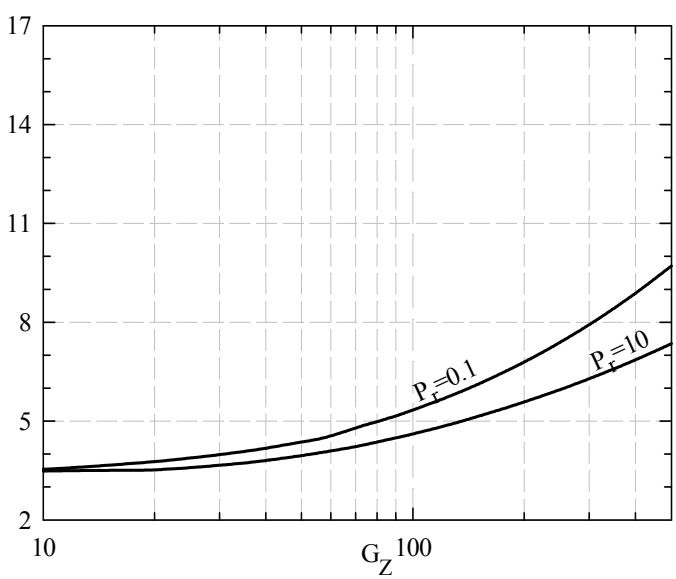

(c) $\mathrm{n}=1.5$

- Present work

$\diamond \quad$ Sayed-Ahmed [12]

- Etemad [11]

○ Etemad \& Mujumdar [10]

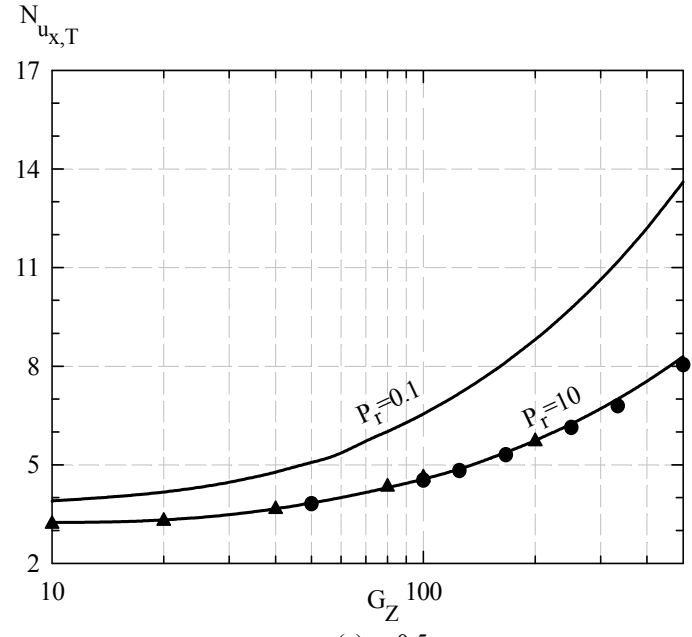

(a) $\mathrm{n}=0.5$

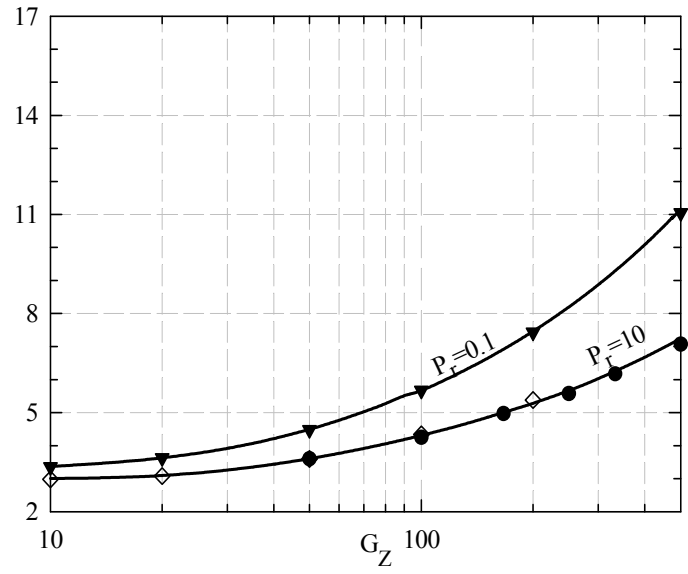

(b) $n=1$

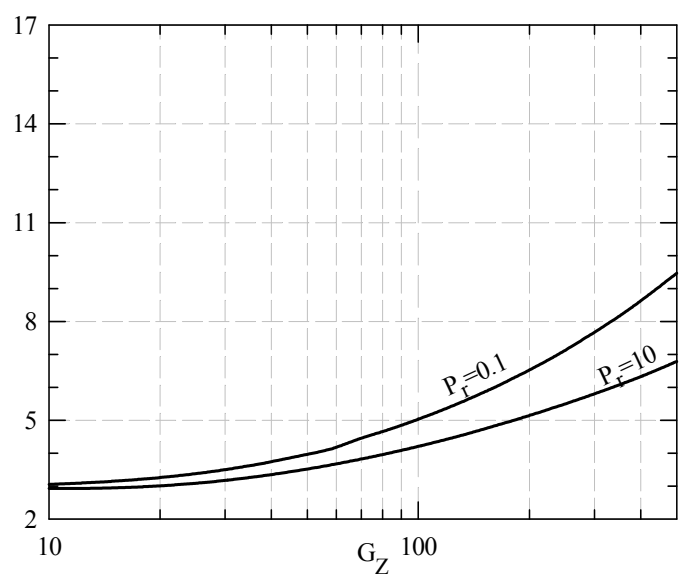

(c) $\mathrm{n}=1.5$
Fig.6. The variation of the local Nusselt number $N u_{x, T}$ with Graetz number $G_{z}$ for case (i), $\alpha=0.5, \tau_{D}$ from 0 to 0.6
Fig.7. The variation of the local Nusselt number $N u_{x, \tau}$ with Graetz number $G_{z}$ for case (i), $\alpha=1, \tau_{D}$ from 0 to 0.6 


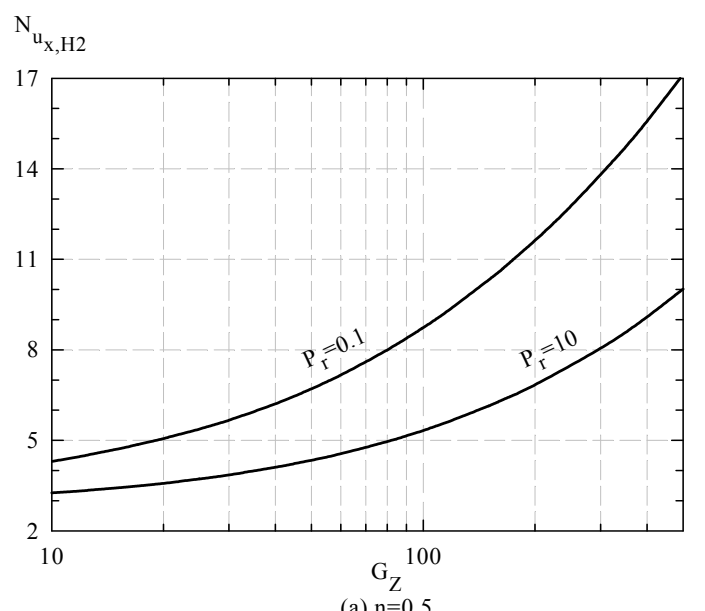

(a) $\mathrm{n}=0.5$

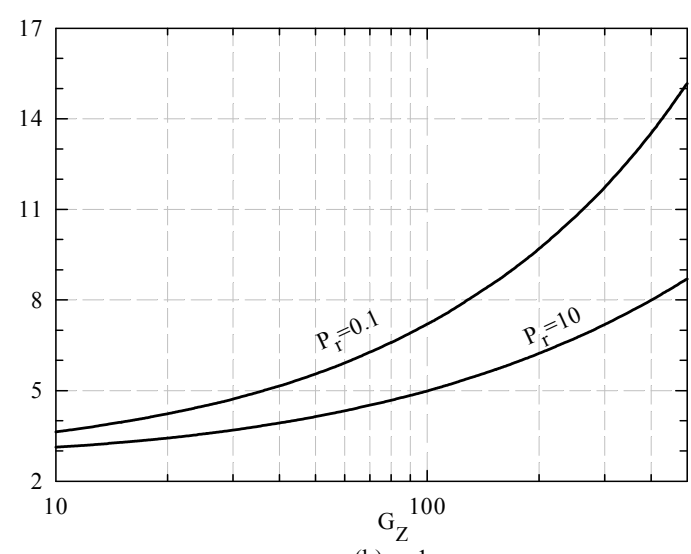

(b) $n=1$

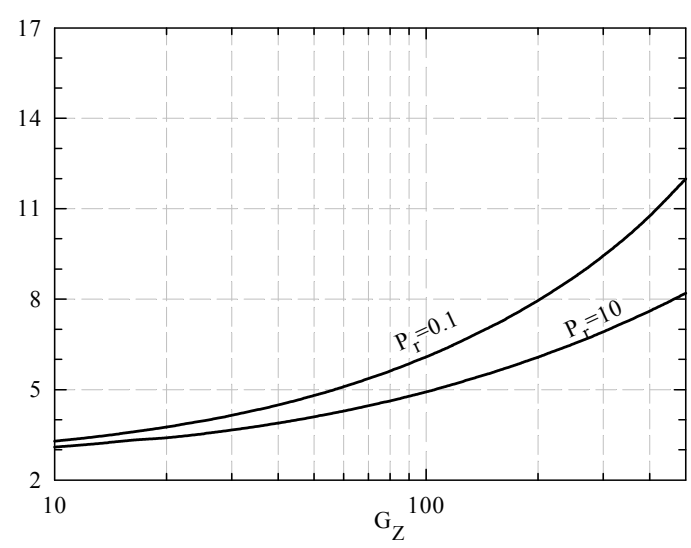

(c) $\mathrm{n}=1.5$

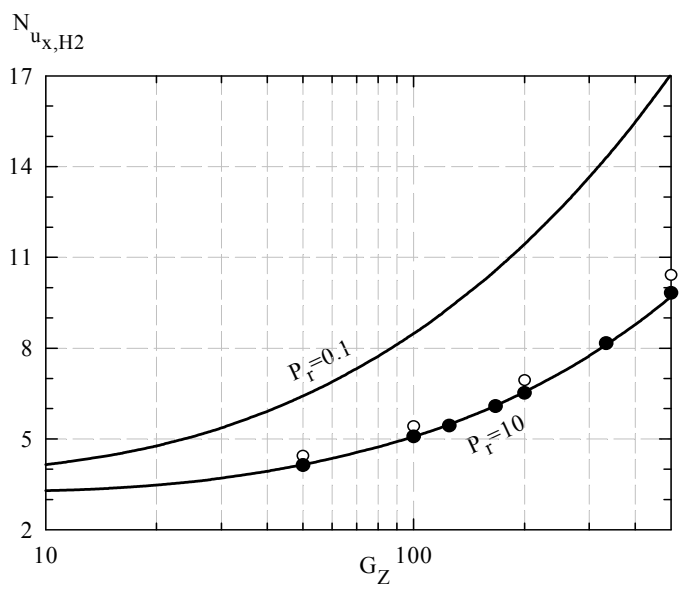

(a) $\mathrm{n}=0.5$

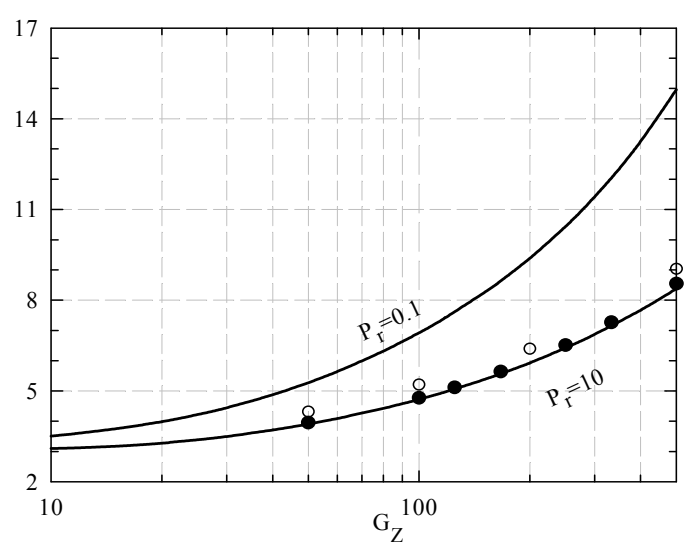

(b) $n=1$

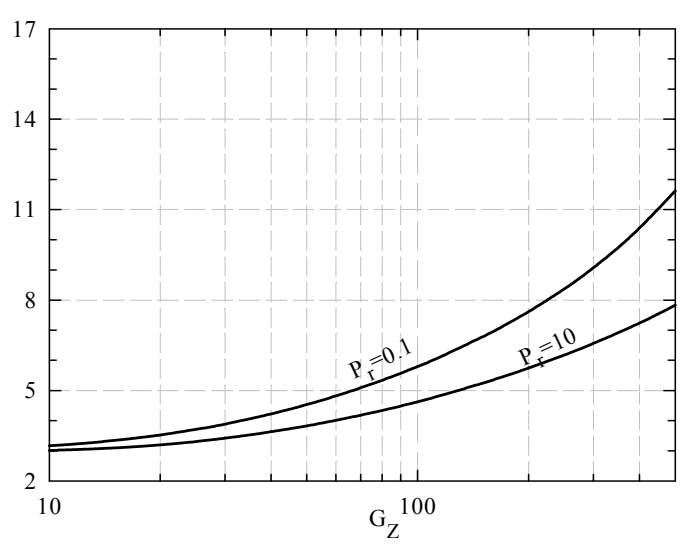

(c) $\mathrm{n}=1.5$

— Present work $\quad \circ$ Etemad \& Mujumdar [10] $\quad$ Etemad [11]

Fig.8. The variation of the local Nusselt number $N u_{x, H 2}$ with Graetz number $G_{Z}$ for case (ii), $\alpha=0.5, \tau_{D}$ and from 0 to 06
Fig.9. The variation of the local Nusselt number $N u_{x, H 2}$ with Graetz number $G_{Z}$ for case (ii), $\alpha=1$ and $\tau_{D}$ from 0 to 0.6 


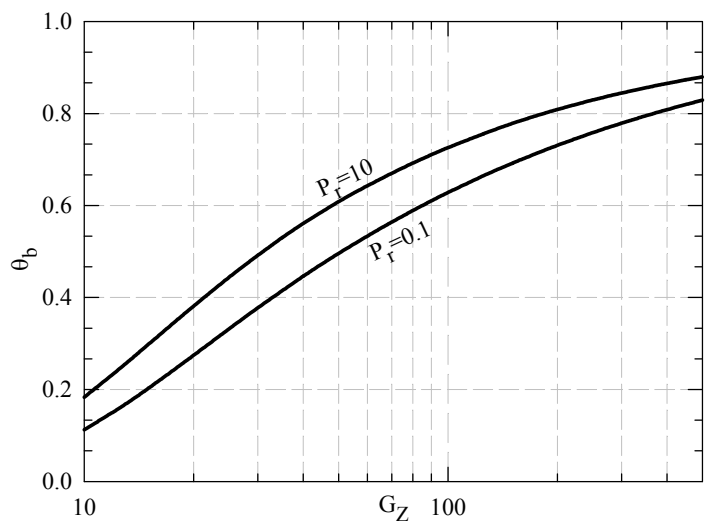

(a) $\mathrm{n}=0.5$

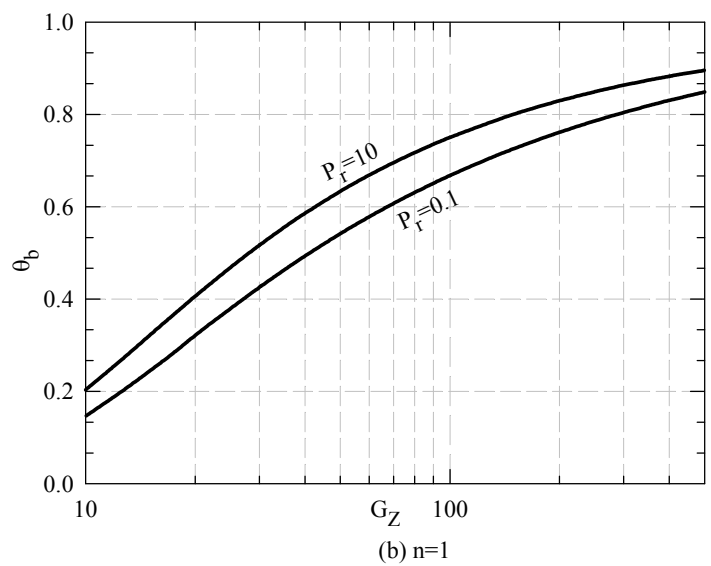

(b) $n=1$

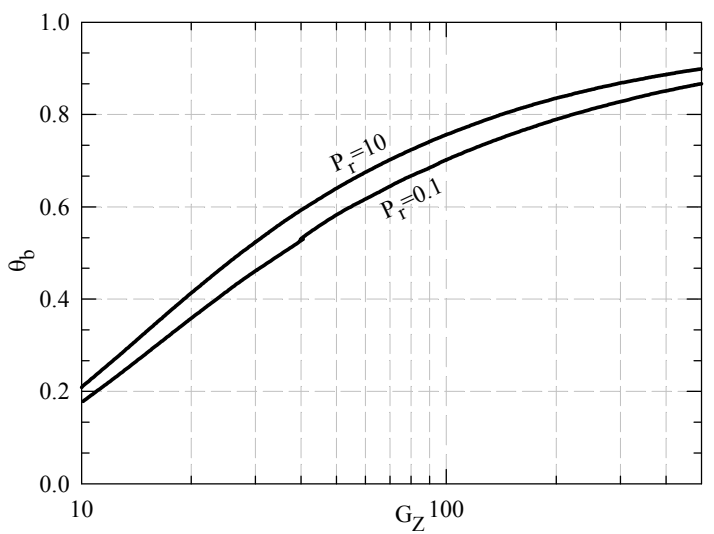

(c) $\mathrm{n}=1.5$

Fig.10. The variation of the bulk mean temperature $\theta_{b}$ with the Graetz number $G_{z}$ for case (i), $\alpha=0.5, \tau_{D}$ from 0 to 0.6

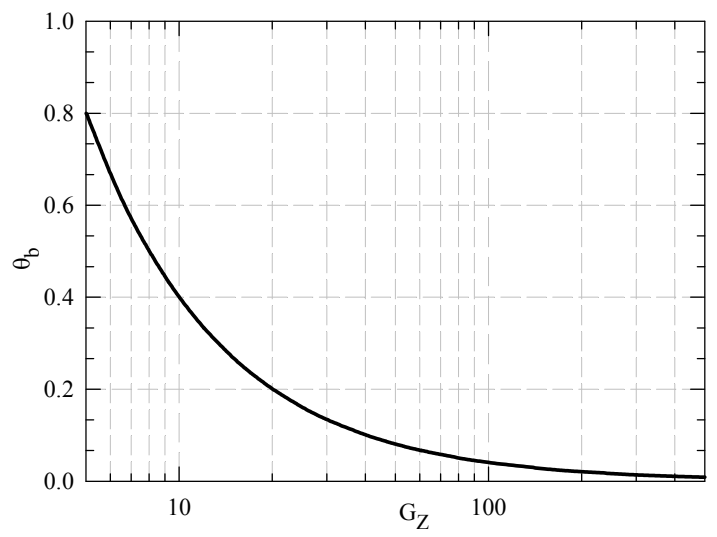

Fig.11. The variation of the bulk mean temperature $\theta_{b}$ with the Graetz number $G_{z}$ for case (ii) 